\title{
Global ray-casting range image registration
}

\author{
Linh $\mathrm{TaO}^{1 *}$, Tam Bui ${ }^{2}$ and Hiroshi Hasegawa ${ }^{3}$
}

\begin{abstract}
This paper presents a novel method for pair-wise range image registration, a backbone task in world modeling, parts inspection and manufacture, object recognition, pose estimation, robotic navigation, and reverse engineering. The method finds the most suitable homogeneous transformation matrix between two constructed range images to create a more complete 3D view of a scene. The proposed solution integrates a ray casting-based fitness estimation with a global optimization method called improved self-adaptive differential evolution. This method eliminates the fine registration steps of the well-known iterative closest point (ICP) algorithm used in previously proposed methods, and thus, is the first direct global registration algorithm. With its parallel implementation potential, the ray casting-based algorithm speeds up the fitness calculation for the global optimization method, which effectively exploits the search space to find the best transformation solution. The integration was successfully implemented in a parallel paradigm on a multi-core computer processor to solve a simultaneous 3D localization problem. The fast, accurate, and robust results show that the proposed algorithm significantly improves on the registration problem over state-of-the-art algorithms.
\end{abstract}

Keywords: Range image registration, Direct global registration, Adaptive differential evolution, Global optimization, Ray-casting, 3D localization

\section{Introduction}

The introduction of commercial depth sensing devices, such as the Microsoft Kinect and Asus Xtion, has shifted the research areas of robotics and computer vision from 2D-based imaging and laser scanning toward 3D-based depth scenes for environment processing. As physical objects or scenarios are built using more than a single image, images from different times and positions need to be aligned with each other to provide a more complete view. We call the alignment process registration, and it plays a key role in object reconstruction, scene mapping, and robot localization applications. Depending on the number of views that are processed simultaneously, registration is divided into multi-view [1] and pair-wise cases [2]. Our paper focuses on the latter case for constructed range images captured by 3D cameras. From two images, called the model and the data, the registration algorithm finds the best homogeneous transformation that aligns

\footnotetext{
*Correspondence: nb14505@shibaura-it.ac.jp

'Department of Functional Control System, Shibaura Institute of Technology,

307 Fukasaku, Minuma-ku, Saitama City, Saitama, 337-8570, Japan

Full list of author information is available at the end of the article
}

the data and the model image in a common coordinate system.

The iterative closest point (ICP) algorithm [3] and its variants, such as EM-ICP [4] and generalized ICP [5], have been indispensable tools in registration algorithms. ICP's concept and implementation are easy to understand. It derives a transformation that draws images closer to each other using their $L_{2}$ error iteratively. ICP-class algorithms have a drawback for general registration in that they require a further assumption of near-optimal initial pose transformation; otherwise, the registration process is likely to converge to local instead of global or near global optima. Some mesh and point cloud editor software programs, such as Meshlab [6], include an ICP built-in registration tool; however, they require that users perform manual pre-alignment before ICP can be applied.

To overcome the shortage of ICP-class methods, automatic registration algorithms in general perform two steps: coarse initialization and fine transformation. If two point clouds are sufficiently close, the first step can be omitted. Otherwise, researchers are faced with

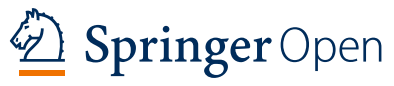

(c) The Author(s). 2017 Open Access This article is distributed under the terms of the Creative Commons Attribution 4.0 International License (http://creativecommons.org/licenses/by/4.0/), which permits unrestricted use, distribution, and reproduction in any medium, provided you give appropriate credit to the original author(s) and the source, provide a link to the Creative Commons license, and indicate if changes were made. 
a big challenge. Two approaches for coarse transformation, pre-alignment estimation, or initialization exist: local and global. The former uses local descriptors (or signatures), such as PFH [7] and SIFT [8], which encode local shape variation in neighborhood points. If the key points of these descriptors appear in both registered point clouds, the initialization movement can be estimated by using sample consensus algorithms, such as RANSAC [9]. Unfortunately, it is not always guaranteed that these signatures will appear in both registered point clouds. On the other hand, global approaches, such as Go-ICP [10] and SAICP [11], take all the points into account. The computation cost is the biggest problem in this approach. In big number data cases, the computation cost becomes large. By virtue of new search algorithms, in particular heuristic optimal methods, and the increase in computer speed achieved by using multi-core computer processor units (CPUs) and graphic computation units (GPUs) [12], it is possible to find reasonable solutions using global approaches for the registration problem. When the coarse transformation has been estimated, the ICP algorithm is an efficient tool for finding the fine transformation.

By integrating optimal search tools with an ICP algorithm, researchers have created hybrid algorithms that integrate global optimizers with ICP. However, this approach has its limitations. SAICP, a parameter-based algorithm, uses simulated annealing (SA) [13] as a search engine to find the best movement combination of rotation angles and translation. However, SA is not sufficiently effective to allow its application to a complicated fitness function, where the potential of a failed convergence is high. Go-ICP converges slowly, since it uses the branchand-bound $(\mathrm{BnB})$ method, a time consuming and nonheuristic method, as a search algorithm to ensure a 100\% convergence rate. In addition, ICP algorithms frequently include a kd-tree structure for searching corresponding points. Using the kd-tree nearest neighbor search method also leads to a high computation cost and a long runtime.

In this paper, a new global direct registration method for 3D constructed surfaces captured by range cameras in cases where the initialization is not good is proposed.

- It eliminates the ICP algorithm from the registration process and thus becomes a direct method.

- As other global registration methods, the new method requires no local descriptors and operates directly on raw scanning data.

- The method uses the improved self-adaptive differential evolution (ISADE) algorithm [14] as a search engine to find the global minima as a direct method that does not use a fine registration procedure such as ICP.

- Furthermore, ray casting-based error calculation reduces the computation cost and runtime because of the potential for using parallelized computation. CPU-based parallel computing procedures allow the algorithm to find the solution at a rate equivalent to the online rate.

The structure of this paper is as follows.

- Section 1 comprises the introduction.

- In Section 2, the classic and up-to-date methods of range image registration are presented.

- In Section 3, the methodology and the new approach of the proposed method are provided.

- In Section 4, the experiments and results are described.

- In Section 5, the discussion and conclusions are presented.

\section{Range image registration}

This part summarises some approaches for global range image registration problem up to date.

\subsection{Registration error function and ICP approach}

SVD and PCA [15] are integrated with ICP in classical methods and global search algorithms are integrated with ICP in most current hybrid methods. In this integration, SVD and PCA find the coarse transformation while ICP is the fine transformation estimation tool. The original version of the ICP algorithm relies on the $L_{2}$ error to derive the transformation (rotation $\mathbf{R} \in S O^{3}$ and translation $\mathbf{t} \in R^{3}$ ), which minimizes the $L_{2}$ type error:

$$
\mathbf{E}(\mathbf{R}, \mathbf{t})=\sum_{\mathbf{i}=\mathbf{1}}^{\mathbf{n}} \mathbf{e}_{\mathbf{i}}(\mathbf{R}, \mathbf{t})=\sum_{\mathbf{i}=\mathbf{1}}^{\mathbf{n}}\left|\mathbf{R} \mathbf{x}_{\mathbf{i}}+\mathbf{t}-\mathbf{y}_{\mathbf{j}^{*}}\right|
$$

where $X=\left\{x_{i}\right\},\{i=1,2,3, \ldots, m\}$ is the model point cloud and $Y=\left\{y_{j}\right\},\{j=1,2,3, \ldots, n\}$ is the data point cloud, $x_{i}$ and $y_{j} \in R^{3}$ are the coordinates of the points in the point clouds, $\mathbf{R}$ and $\mathbf{t}$ are the rotation and translation matrix, respectively, $\mathbf{y}_{\mathbf{j}^{*}}$ is the corresponding point of $\mathbf{x}_{\mathbf{i}}$ denoting the closest point in data point cloud $Y$. $\mathbf{R}$ and $\mathbf{t}$ are determined by Roll-Pitch-Yaw movement of three rotation angles $(\alpha, \beta, \gamma)$ and translation values $(x, y, z)$.

Variants of the ICP algorithm rely on different distance categories to define the closest points. Point-to-point distance and point-to-plane distance are two popular examples. Equation 2 presents the former case.

$$
\mathbf{j}^{*}=\underset{\mathbf{j} \in\{\mathbf{1}, \ldots, n\}}{\operatorname{argmin}}\left\|\mathbf{R} \mathbf{x}_{\mathbf{i}}+\mathbf{t}-\mathbf{y}_{\mathbf{j}}\right\|
$$

The following iterative process is designed to achieve the final transformation.

1. Compute the closest model points for each data point as in Eq. 2.

2. Compute the transformation $\mathbf{R}$ and $\mathbf{t}$ based on the error obtained using Eq. 1. 
3. Apply $\mathbf{R}$ and $\mathbf{t}$ to the data point clouds.

4. Repeat steps 1, 2, and 3 until the error obtained using (1) is smaller than a set tolerance level or the procedure reaches its maximum iteration.

Step by step, the data point cloud becomes closer to the model point cloud and the process stops at local minima. ICP's variants, such as LMICP [16] and SICP [17], use different methods to calculate the transformation from error $\mathbf{E}(\mathbf{R}, \mathbf{t})$. A well-known accumulation registration method in the KinectFusion algorithm [18] uses ICP to register two consecutive frames. The transformation matrix for the current frame is estimated by multiplying the matrices from the previous registration steps.

\subsection{Global hybrid registration algorithm}

ICP algorithms constitute the most suitable method for registering close or pre-aligned point cloud data. In other cases, the algorithm frequently converges incorrectly. Global search algorithms are suitable for solving this problem, since they can find the global instead of the local minima. To reduce the burden of the global search algorithm, researchers frequently flatten the search space by using ICP. Figures 1 and 2 show an example of ICP's operation as a flattening tool. In Fig. 1, from any beginning point, after many iterations, ICP finds the nearest local optima point. Figure 2 shows that a complex fitness function (colored black) becomes a simpler one (colored red). As a result, global search methods are able to find the global minima more effectively.

The integration is effective in the case of point cloud data where the point number is small. For cases where the point number is large, the hybrid approach with ICP becomes slow. This method cannot therefore be implemented in real-time applications.

\section{The new direct global approach}

With the newly developed global search algorithms, flattening using ICP inner loops in registration becomes redundant. Our method integrates a new global search algorithm, ISADE, which is suitable for complicated fitness functions when the flattening process is not performed, and a ray casting-based corresponding search method to accelerate the objective function calculation in the registration procedure.

\subsection{Ray-casting for fast corresponding point determination on constructed range image}

The KinectFusion algorithm, a real-time scene reconstructing pipeline, uses ICP as the only method for registering two continuous frames. The procedure requires a powerful GPU to speed up calculations and reduce runtime. However, global registration algorithms calculate a thousand times more error functions than ICP and thus, so that these algorithms can be applied online or using less powerful processors, faster error calculation methods must be included.

ICP algorithms use the kd-tree [19] structure to speed up the process of determining $j^{*}$ in Eq. 2. The complexity of the kd-tree nearest neighbor search algorithms is $O(\log (n))$, where $n$ is the set number of the search points. Figure 3 shows an example of the true closest corresponding points of the model and data point clouds.

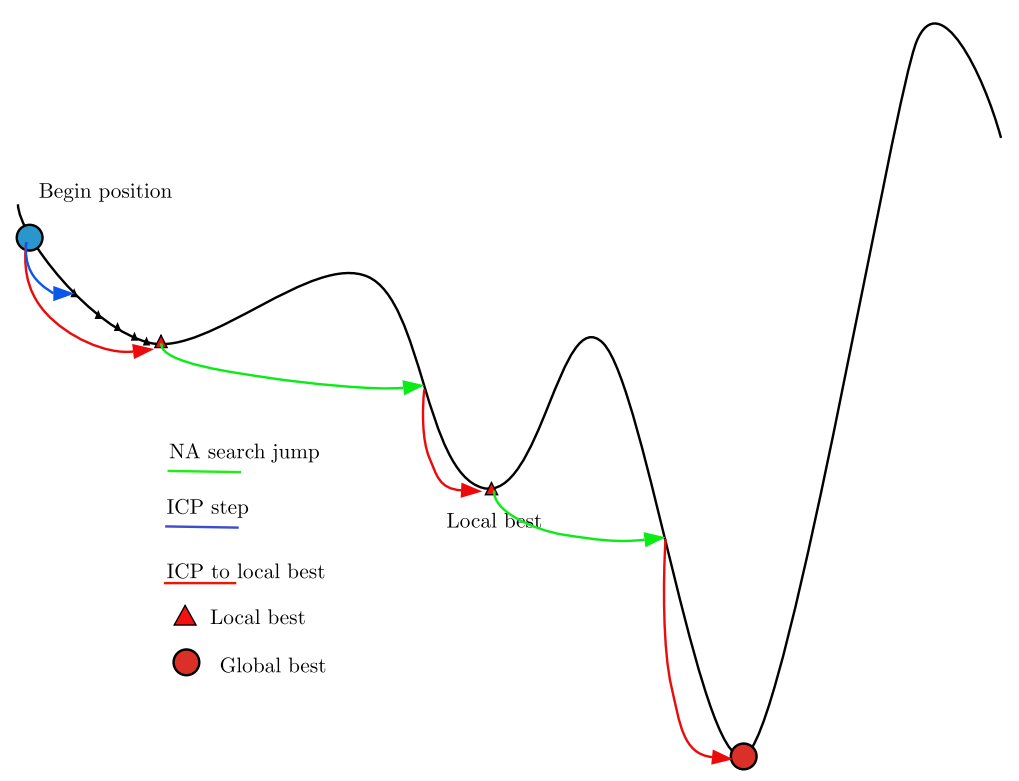

Fig. 1 Global searching algorithm with ICP integrated 


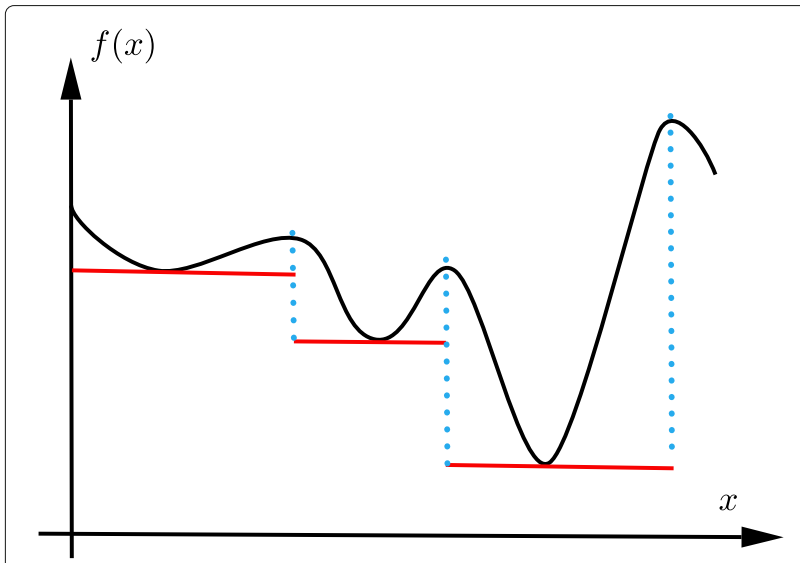

Fig. 2 Example of flatten objective function after ICP in red color where original function is in black

Ray casting [20] is one of the most basic of the many computer graphics rendering methods. The idea behind the ray-casting method is to direct a ray from the eye through each pixel and find the closest object blocking the path of the ray. Using the material properties and light effect in the scene, rendering methods can determine the shading of the object. Some hidden surface removal algorithms use ray casting to find the closest surfaces to the eye and eliminate all others that are at a greater distance along the same ray. The Point Cloud Library [21] uses ray casting as a filtering method; it removes all points that are obscured by other points.

We apply ray casting to find the approximated closest point using a range camera model. Constructed range images or point cloud data are frequently captured by a $3 \mathrm{D}$ range camera, where a range image can be considered a $2 D$ gray image, $G$; the value of each pixel shows the depth of a point. To simplify the problem, we do not take distortion into consideration.

$$
z_{i, j}=G_{i, j}
$$

where $z_{i, j}$ is the depth of the image at pixel column $i$ and row $j$.

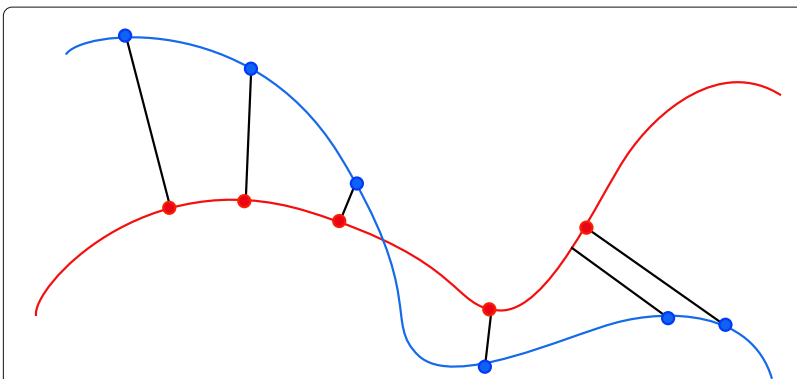

Fig. 3 Closest corresponding point using kd-tree. Data points are in blue and model points are in red
Equation 4 converts range image data points to real 3D depth data $\{x, y, z\}$ in $R^{3}$.

$$
\begin{aligned}
& x_{i, j}=(i-c x) G_{i, j} / f x \\
& y_{i, j}=(j-c y) G_{i, j} / f y \\
& z_{i, j}=G_{i, j}
\end{aligned}
$$

where $f x, f y, c x$, and $c y$ are the intrinsic parameters of the depth camera.

Inversely, pixel position $i, j$ is to be calculated. Figure 4 shows the method's idea.

Using the corresponding points obtained in the raycasting step, we determine the depth difference $\Delta z_{i, j}$ for the next step of calculating the objective function for the global search method, as

$\Delta z_{i, j}(R, t)=\left\{\begin{array}{cc}z_{i, j}^{X}-z_{i, j}^{Y, t} & \text { if } \\ 0 & \text { otherwise }\end{array}\right\}$

where $R$ and $t$ are the rotation and translation matrix, respectively, $z_{i, j}^{X}$ is the depth of the model point cloud, and $z_{i, j}^{Y(R, t)}$ is the depth of the data point cloud after applying the rotation and translation matrix with $i, j$ from the ray casting process.

The ray-casting method is simple and fast (with a complexity of $O(1)$ ) and, more importantly, potentially parallel computing can be applied.

\subsection{Objective function}

Global optimization methods use fitness or objective functions to find the transformation that drives the fitness function to the smallest value. We propose a fitness function $F(R, t)$ :

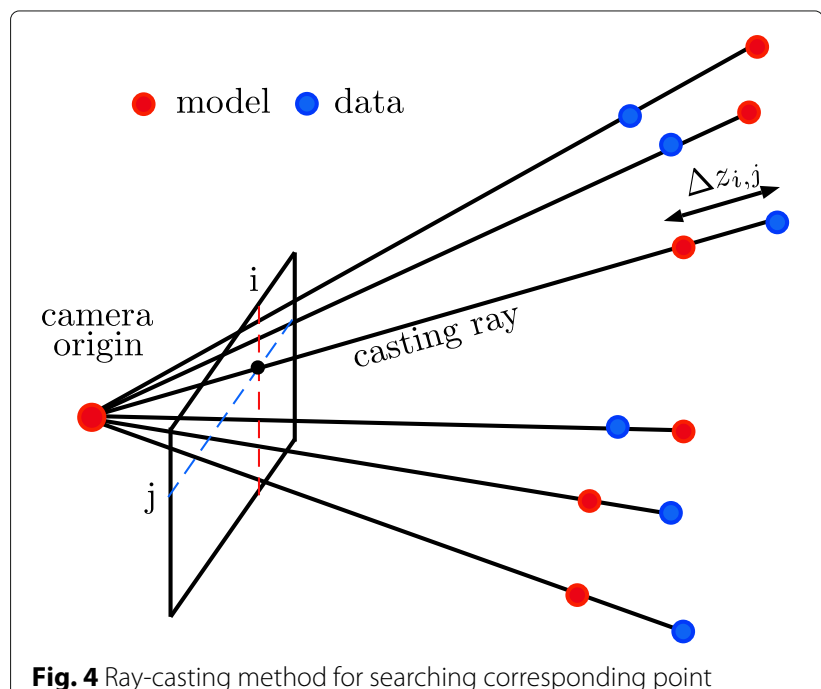




$$
\mathbf{F}(\mathbf{R}, \mathbf{t})=\mathbf{f}(\mathbf{k}) \frac{\mathbf{1}}{\mathbf{k}^{2}} \sum_{\mathbf{i}=\mathbf{1}}^{\mathbf{n}} \sum_{\mathbf{j}=\mathbf{1}}^{\mathbf{m}}\left(\Delta \mathbf{z}_{\mathbf{i}, \mathbf{j}}(\mathbf{R}, \mathbf{t})\right)^{2}
$$

where $R$ and $t$ are the rotation and translation matrix, respectively, $m$ and $n$ are the height and width of the image frame, and $k$ is the inlier point number.

To gain a smaller error in a larger number of inlier points, we used an additional function $f(k)$ :

$$
\mathbf{f}(k)=\left\{\begin{array}{cc}
\infty & \text { if } k<N / 10 \\
1-k / N & k \geqslant N / 10
\end{array}\right\}
$$

where $N$ is the number of points in the data point cloud.

The ray-casting-based method makes the algorithm run significantly faster than the kd-tree-based approach. However, since a global search algorithm handles a large number of points at a huge computation cost, we take parallel implementation into consideration. Since in most computers a multi-core processor is available, using the CPU for parallel computing is convenient in most applications. In addition, CPU multi-core parallel implementation is even easier with OpenMP library [22]. Furthermore, the ray-casting process adapts well to parallel computing, and the corresponding points can be calculated in different processes or threads.

\subsection{ISADE, an efficient improved version of differential evolution algorithm}

\subsubsection{Differential evolution}

Differential evolution (DE) is an evolutionary optimization technique originally proposed by Storn and Price [23], characterized by operators of mutation and crossover. In DE, the scaling factor $F$ and crossover rate $C_{r}$ determine the correction and speed of convergence, while another important parameter, $N P$, the population size, remains a user-assigned parameter to handle problem complexity. Figure 5 shows pseudo-code or implementation flowchart of DE algorithms.

a) Initialization in $D E$ The initial population was generated uniformly at random in the range lower boundary (lb) and upper boundary (ub).

$$
X_{i}^{G}=l b_{j}+\operatorname{rand}_{j}(0,1)\left(u b_{j}-l b_{j}\right)
$$

where $\operatorname{rand}_{j}(0,1)$ a random number $\in[0,1]$.

b) Mutation operation In DE, there are various mutation schemes to create mutant vectors $V_{i}^{G}=\left(V_{i, 1}^{G}, \ldots, V_{i, D}^{G}\right)$ for each individual of population at each generation $G$. $X_{i}^{G}$ is target vector in the current population, $D$ is vector dimension number.

$\mathrm{DE} / \mathrm{rand} / 1: V_{i, j}^{G}=X_{r_{1}, j}^{G}+F\left(X_{r_{2}, j}^{G}-X_{r_{3}, j}^{G}\right)$

$\mathrm{DE} /$ best $/ 1: V_{i, j}^{G}=X_{\text {best }, j}^{G}+F\left(X_{r_{1}, j}^{G}-X_{r_{2}, j}^{G}\right)$

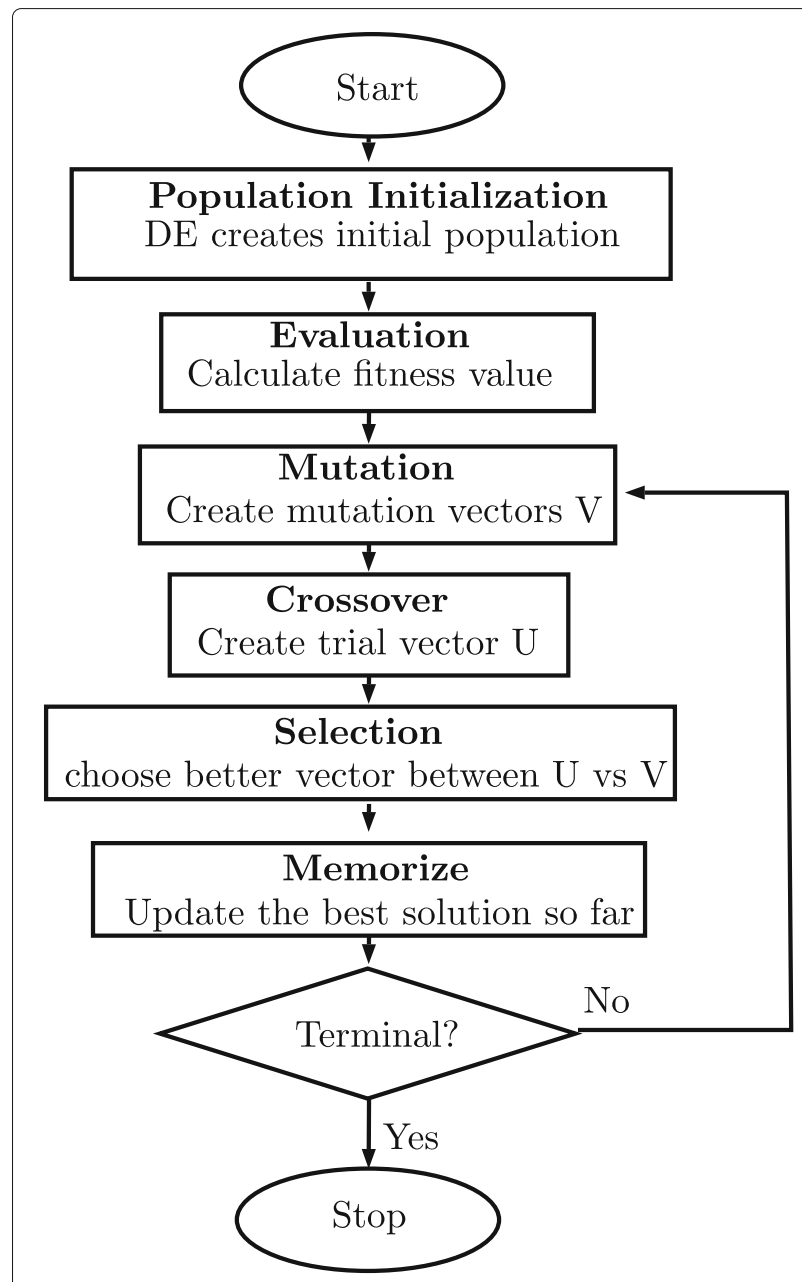

Fig. $5 \mathrm{DE}$ implementation process

$\mathrm{DE} /$ currenttobest $/ 1: V_{i, j}^{G}=X_{i, j}^{G}+F\left(X_{\text {best }, j}^{G}-X_{i, j}^{G}\right)+F\left(X_{r_{b} j}^{G}-X_{r_{2}, j}^{G}\right)$

$\mathrm{DE} / \mathrm{rand} / 2: V_{i, j}^{G}=X_{i, j}^{G}+F\left(X_{r_{2}, j}^{G}-X_{r_{3}, j}^{G}\right)+F\left(X_{r_{4}, j}^{G}-X_{r_{5}, j}^{G}\right)$

$\mathrm{DE} /$ best $/ 2: V_{i, j}^{G}=X_{\text {best }, j}^{G}+F\left(X_{r 1, j}^{G}-X_{r 2, j}^{G}\right)+F\left(X_{r 3, j}^{G}-X_{r 4, j}^{G}\right)$

DE/randtobest $/ 1: V_{i, j}^{G}=X_{\text {best }, j}^{G}+F\left(X_{\text {best }, j}^{G}-X_{r 2, j}^{G}\right)+F\left(X_{r 2, j}^{G}-X_{r 3, j}^{G}\right)$

where $r_{1}, r_{2}, r_{3}, r_{4}$, and $r_{5}$ are randomly selected integers in the range $[1, N P]$. 
c) Crossover operation After mutation process, DE performs a binomial crossover operator on $X_{i}^{G}$ and $V_{i}^{G}$ to generate a trial vector $U_{i}^{G}=\left(U_{i, 1}^{G}, \ldots, U_{i, D}^{G}\right)$ for each individual population $i$ as shown in Eq. 10.

$$
U_{i, j}^{G}=\left\{\begin{array}{ccc}
V_{i, j}^{G} & \text { if } \quad \operatorname{rand}_{j} \leqslant C_{r} \text { or } j=j_{\text {rand }} \\
X_{i, j}^{G} & \text { otherwise }
\end{array}\right\}
$$

where $i=1, \ldots, N P, \quad j=1, \ldots, D, j_{\text {rand }}$ is a randomly chosen integer in $[1, D], \operatorname{rand}_{j}(0,1)$ is a uniformly distributed random number between 0 and 1 generated for each $j$ and $C_{r} \in[0,1]$ is called the crossover control parameter. Using $j_{\text {rand }}$ ensures the difference between the trial vector $U_{i}^{G}$ and target vector $X_{i}^{G}$.

c) Selection operation The selection operator is performed to select the better solution between the target vector $X_{i}^{G}$ and the trial vector $U_{i}^{G}$ entering to the next generation.

$$
X_{i}^{G+1}=\left\{\begin{array}{lll}
U_{i}^{G} & \text { if } & f\left(U_{i}^{G}\right) \leqslant f\left(X_{i}^{G}\right) \\
X_{i}^{G} & \text { otherwise }
\end{array}\right\}
$$

where $i=1, \ldots, N P, X_{i}^{G+1}$ is a target vector in the next generation's population.

\subsubsection{Improvement of self-adapting control parameters in differential evolution}

a) Adaptive selection learning strategy in mutation operator In our study of ISADE, we randomly chose three mutation schemes: DE/best/1/bin, DE/best/2/bin, and DE/rand to best $/ 1 /$ bin. $D E /$ best $/ 1 /$ bin and $D E /$ best $/ 2 /$ bin have a good convergence property, and $\mathrm{DE} / \mathrm{rand}$ to best/1/bin has a good population diverse property. The probability of applying these strategies is equal at values of $p_{1}=p_{2}=$ $p_{3}=1 / 3$.

DE/best $/ 1: V_{i, j}^{G}=X_{\text {best }, j}^{G}+F\left(X_{r_{1}, j}^{G}-X_{r_{2}, j}^{G}\right)$

DE/best $/ 2: V_{i, j}^{G}=X_{\text {best }, j}^{G}+F\left(X_{r 1, j}^{G}-X_{r 2, j}^{G}\right)+F\left(X_{r 3, j}^{G}-X_{r 4, j}^{G}\right)$

DE/randtobest $/ 1: V_{i, j}^{G}=X_{\text {best }, j}^{G}+F\left(X_{\text {best }, j}^{G}-X_{r 2, j}^{G}\right)+F\left(X_{r 2, j}^{G}-X_{r 3, j}^{G}\right)$

where $r_{1}, r_{2}, r_{3}, r_{4}$, and $r_{5}$ are randomly selected integers in the range $[1, N P]$, where $N P$ is the population size.

b) Adaptive scaling factor To achieve a better performance, ISADE gives the scale factor $F$ a large value initially to allow better exploration and a small value after the generations to allow appropriate exploitation. Instead of using sigmoid scaling in Eq. 13 taken from Tooyama and Hasegawa's study on APGA/VNC [24], ISADE adds a new factor to calculate $F$ as shown in Eq. 14.

$$
\begin{aligned}
F_{i} & =\frac{1}{1+\exp \left(\alpha * \frac{i-N P / 2}{N P}\right)} \\
F_{i} & =\frac{F_{i}+F_{i}^{\text {mean }}}{2}
\end{aligned}
$$

in which $F_{i}^{\text {mean }}$ is calculated as Eq. 15 .

$$
F_{i}^{\text {mean }}=F_{\min }+\left(F_{\max }-F_{\min }\right)\left(\frac{i_{\max }-i}{i_{\max }}\right)^{n_{\mathrm{iter}}}
$$

where $F_{\max }$ and $F_{\min }$ denote the lower and upper boundary condition of $F$ with recommended values of 0.8 and 0.15 , respectively. $i, i_{\max }$, and $n_{\text {iter }}$ denote the current, max generation, and nonlinear modulation index as in Eq. 15.

$$
n_{\text {iter }}=n_{\min }+\left(n_{\max }-n_{\min }\right)\left(\frac{i}{i_{\max }}\right)
$$

where $n_{\max }$ and $n_{\min }$ are typically chosen in the range $[0,15]$. Recommended values for $n_{\min }$ and $n_{\max }$ are 0.2 and 6.0 respectively.

c) Crossver control parameter ISADE is able to detect whether the height of $C_{r}$ values are useful. The control parameter $C_{r}$ is assigned as

$$
C_{r}^{i+1}=\left\{\begin{array}{cc}
\operatorname{rand}_{2} & \text { if } \operatorname{rand}_{1} \leqslant \tau \\
C_{r}^{i} & \text { otherwise }
\end{array}\right\}
$$

where $\operatorname{rand}_{1}$ and rand $_{2}$ are random values $\in[0,1], \tau$ represents the probability to adjust $C_{r}$, which is also updated using

$$
C_{r}^{i+1}=\left\{\begin{array}{ll}
C_{r_{\min }} & C_{r_{\text {min }}} \leqslant C_{r}^{i+1} \leqslant C_{r_{\text {medium }}} \\
C_{r_{\max }} & C_{r_{\text {medium }}} \leqslant C_{r}^{i+1} \leqslant C_{r_{\max }}
\end{array}\right\}
$$

where $C_{r_{\text {min }}}, C_{r_{\text {medium }}}$, and $C_{r_{\max }}$ denote a low value, median value, and high value of the crossover parameter, respectively. We use recommended values of $\tau=0.1, C_{r_{\min }}=$ $0.05, C_{r_{\text {medium }}}=0.50$, and $C_{r_{\max }}=0.95$.

d) Combination of ISADE and ray-casting ISADE eliminates tuning tasks for the problem-dependent parameters $F$ and $C_{r}$. With simple adaptive rules, the computation complexity of this new version of the DE algorithm remains the same as that of the original version. All the above ideas and theories of ISADE algorithm and ray-casting method are implemented as in the flowchart shown in Fig. 6.

\section{Experiment and results}

This section describes experiments that were conducted using the proposed method in real range image data registration and presents the results. We integrated different global search methods with the ray casting-based algorithm in order to obtain a comparison between ISADE and the state-of-the-art methods as follows.

1) SA proposed in Luck et al.'s paper, Registration of range data using a hybrid simulated annealing and iterative closest point algorithm. 


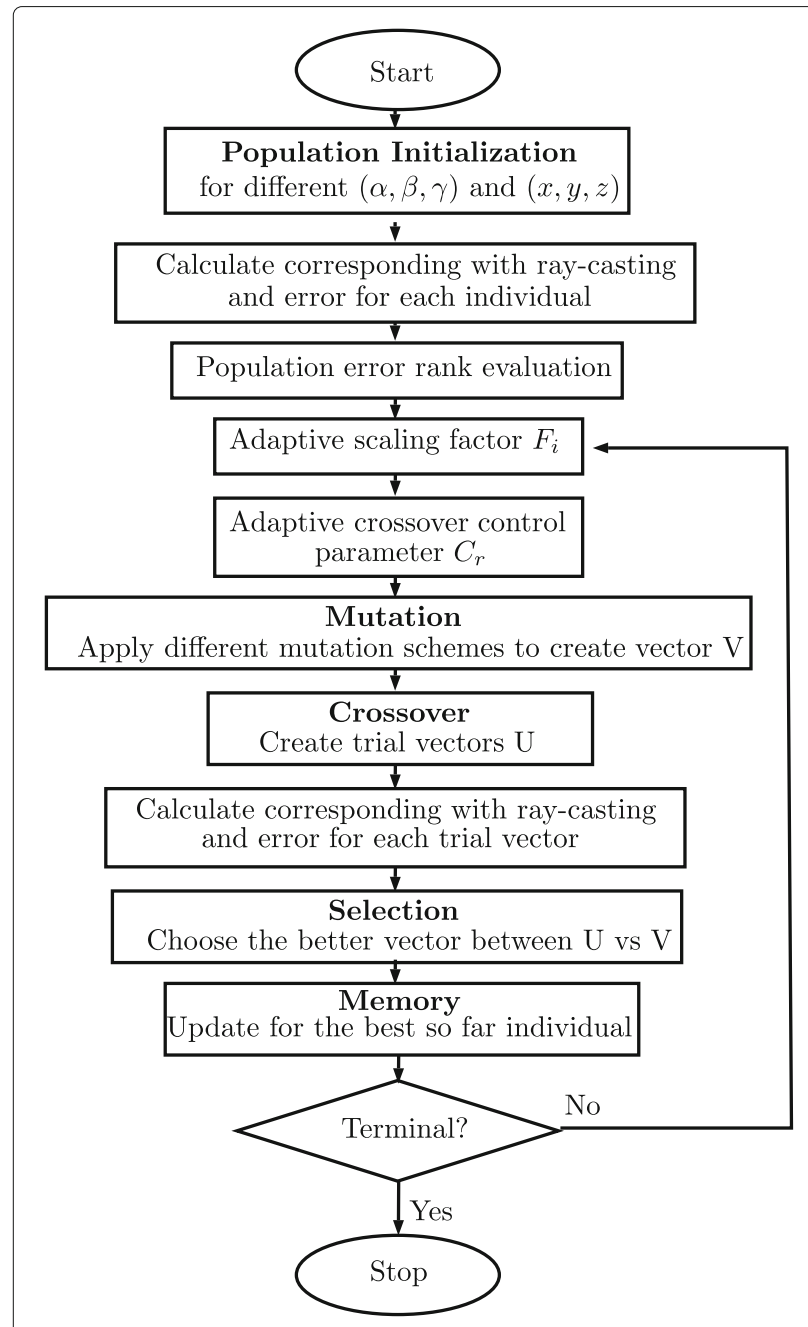

Fig. 6 ISADE implementation process with ray-casting corresponding method

2) Particle swarm optimization (PSO) proposed in Talbi et al.'s paper, Particle swarm optimization for image processing [25].

3) Genetic algorithm (GA) proposed in Valsecchi et al.'s paper, An image registration approach using genetic algorithms [26].

4) DE proposed in Falco et al.'s paper, Differential evolution as a viable tool for satellite image registration [27].

We also calculated the ray casting-based error of the KinectFusion and Go-ICP algorithms for further comparison. All algorithms were implemented in $\mathrm{C}++$ and compiled with GNU/g++ tool.

\subsection{Range image dataset}

In our experiments, a number of pair-wise registrations was conducted using well-known depth data,
"RGB-D Dataset 7-Scenes," taken from the Kinect Microsoft Camera downloaded from the Microsoft Research Website, http://research.microsoft.com/en-us/ projects/7-scenes/. Specifically, Figs. 7 and 8 show all the scenes: Chess, Fire, Heads, Office, Pumpkin, RedKitchen, and Stairs. The details of the data used in the registration experiments are as follows.

Chess dataset: image sequence 2, frame 960 vs frame 980.

Other datasets: image sequence 1 , frame 000 vs frame 020 .

These "PNG" format depth images are sub-sampled into a smaller resolution of $128 \times 96$, which is five times smaller than the original resolution of $640 \times 480$ in each dimension. The purpose of using a dataset with a smaller number of points is to achieve a suitable runtime while preserving robustness and accuracy.

\subsection{Parameter settings}

For each method, 30 runs were performed. The search space had rotation angles and translation limited at $[-\pi / 5, \pi / 5]$ and $[-1,1]$ separately. This means that the limitation of the rotation angles was $36^{\circ}$ and of the translation was $1 \mathrm{~m}$.

The algorithm parameters shown in Table 1 constitute the configuration for all the algorithms. All methods were run on a desktop PC powered with an Intel core I7-4790 CPU $3.60 \mathrm{GHz} \times 8$ processor, $8 \mathrm{~GB}$ RAM memory and Linux Ubuntu 14.04 64-bit Operation System. The new algorithm $\mathrm{C}++$ code was written based on reference from Andreas Geiger's LIBICP code [28].

\subsection{Comparison with KinectFusion algorithm}

Accompanied by depth ranger images, "RGB-D Dataset 7-Scenes" provides homogeneous camera to world transposes at each frame calculated using the KinectFusion algorithm. We converted those camera transposes into transformation matrix between two frames as

$$
\begin{aligned}
& T_{i}^{j}=T_{i}^{-1} * T_{j} \\
& T_{i}^{j}=\left[\begin{array}{lll|l}
R_{i}^{j} & t_{i}^{j} \\
& & & \\
0 & 0 & 0 & 1
\end{array}\right]
\end{aligned}
$$

where $T_{i}^{j}$ is the transformation matrix to move frame $j$ to align with frame $i, T_{i}$ and $T_{j}$ are the homogeneous transpose matrix for the camera at frame $i$ and $j$, respectively, and $R_{i}^{j}$ and $t_{i}^{j}$ are the rotation and translation matrix of $T_{i}^{j}$, respectively. 


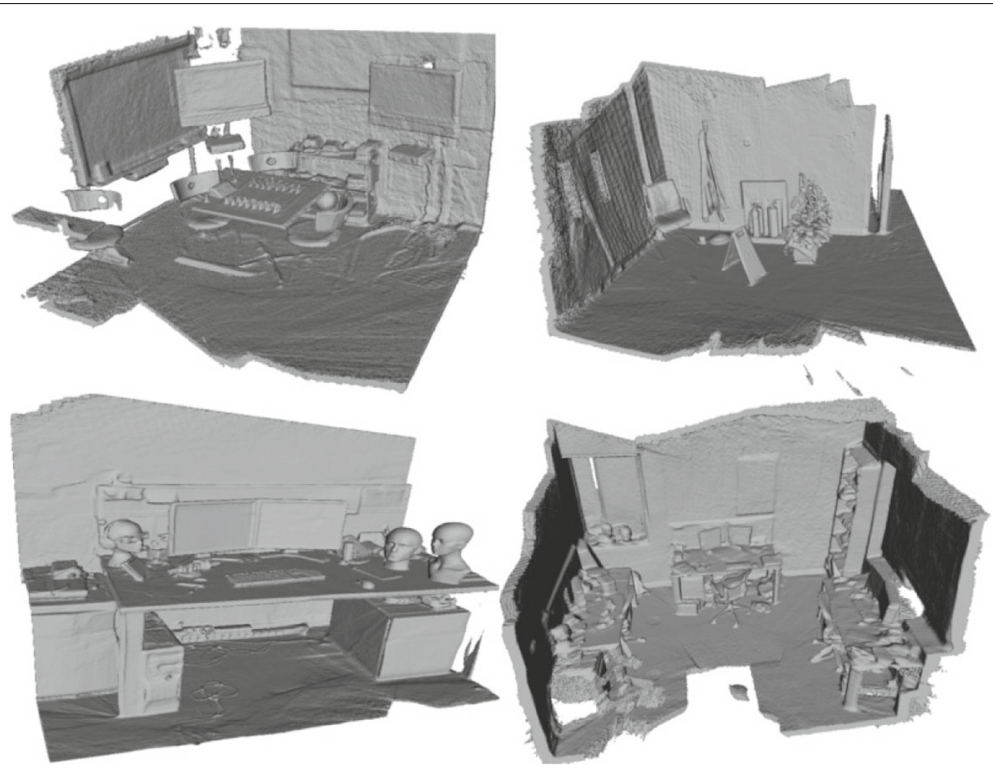

Fig. 7 RGB-D Chess, Fire, Heads, Office dataset for experiments

$R_{i}^{j}$ and $t_{i}^{j}$ are applied to ray-casting error calculation methods for two frames, as in Eq. 6, to describe the errors of the KinectFusion algorithm. Table 2 presents the mean errors of the proposed method in comparison with the error of the KinectFusion algorithm. The significantly smaller mean errors of the proposed method prove its superiority to the KinectFusion algorithm registration pipeline.

Figures 9 and 10 visually show the registration results of the proposed algorithm for the seven scenes in center and those of KinectFusion on the left hand side, to provide a visual comparison. The seven scenes included are Chess, Fire, Heads, Office, Pumpkin, RedKitchen, and Stairs. The model point clouds are colored red, and the data point clouds are colored green.

In these figures, the proposed algorithm outperforms KinectFusion is clearly seen. Even in the best case of KinectFusion, such as Stairs or RedKitchen, the overlapping regions, where the two colors are mixed together, are not as clearly seen as in the results of the proposed algorithm.

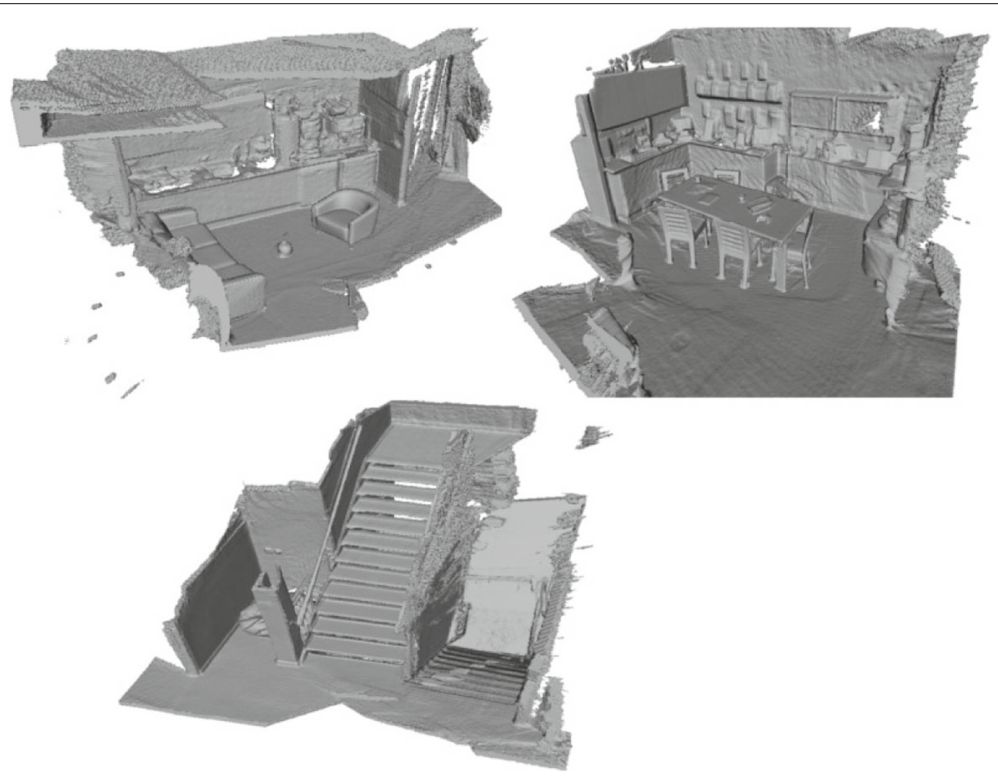

Fig. 8 RGB-D Pumpkins, RedKitchen, Stair dataset for experiments 
Table 1 Algorithm configuration

\begin{tabular}{llllll}
\hline Algorithm & DE & GA & SA & PSO & Go-ICP \\
\hline Parameters & $F_{0}=0.8$ & $P C=0.95 ;$ & $\alpha=0.995$ & elites $=4$ & trimFraction $=0.0$ \\
& $C_{r}=0.9$ & $P m=0.1 ;$ & & neighbors $=5$ & distTransSize $=50$ \\
Maxgen & DE $/$ rand $/ 1 /$ bin & elites $=5$ & & $c 1=c 2=c 3=2.1$ & $100 ;$ \\
Population & 100 & 100 & 3000 & 30 & data subsample $=1000$ points
\end{tabular}

An example of applying the new method to consecutive localizations can be seen in Fig. 11. The pumpkin 3D scene, which is built from seven different range images (frame 000, 020,..., 120), visually shows the accuracy of the proposed method at various percentages of overlapping regions. The different frames are in different colors. A video at https://www.youtube.com/watch?v= sgaUry5qsxU gives a clearer view.

\subsection{Comparison with Go-ICP algorithm}

From authors contributed code [29], we performed experiments to compare our method with Go-ICP on accuracy, runtime, and robustness. Go-ICP configuration parameters were set as in Table 1 with the identical searching boundary with other methods. distTransSize is the number of nodes in translation searching boundary. It was set to 50 or translation resolution is at $40 \mathrm{~mm}$. Raising accuracy by increasing dist TransSize to 500 or $4 \mathrm{~mm}$ resolution effort failed due to infinite runtime. Go-ICP were able to register Heads and Office datasets at distTransSize of 100 with runtime presented in Table 5 .

The disadvantage of big resolution could be compensated by inner ICP loops; however, the smaller the resolution, the more accurate the algorithm is. We set the data subsample to 1000; Go-ICP reaches infinitive runtime at the original $128 \times 96$ resolution.

Together with KinectFusion and our method errors, Table 2 presents the mean errors of Go-ICP algorithm where "nan" stands for undefined result in the case of infinitive runtime and "inf" stands for wrong convergence with few overlapse points. Over all, only heads and office showed good convergence with small error and run time. However, those small errors are still bigger than the new method.
Figures 9 and 10 also show the registration results of GoICP algorithm on the right side together with new method results in the center and KinectFusion algorithm result on the left side. From those figures, the new method better performance is clearly seen. In the case of RedKitchen dataset, the wrong convergence results of Go-ICP were observed, the error was small because of small over-lapsed percentage.

Average runtime for Go-ICP on different datasets are presented in Table 5 where average run times of the new algorithm at different generation numbers are presented. In the table, "inf" values stand for infinitive runtime. GoICP was fast in case of heads dataset or extreme slow for the case of Chess dataset.

Over all, the new methods outperformed Go-ICP on experiment datasets in accuracy, runtime, and robustness.

\subsection{Comparison between different optimization algorithms}

Tables 3 and 4 show the experimental results of all the integrations and methods in four categories: $\min , \max$, mean, and standard deviation.

The smaller means and standard deviations for every dataset in comparison with the other methods show the accuracy and robustness of the new search engine as compared to the state-of-the-art search algorithms. In some cases, the experimental results show that the other integrations performed better than KinectFusion. The ICP accumulating error is the reason for this poor performance.

\subsection{Iterations vs convergence}

In Fig. 12, we compare the robust results of convergence of the registration of the seven scenes for a small number of iterations between using ISADE and

Table 2 Error comparison between new method, KinectFusion, and Go-ICP algorithms

\begin{tabular}{lllllcc}
\hline & Chess & Fire & Heads & Office & Pumpkin & RedKitchen \\
\hline Our method & $\mathbf{0 . 1 0 2 3 0}$ & $\mathbf{0 . 0 3 1 7 9}$ & $\mathbf{0 . 0 1 0 0 0}$ & $\mathbf{0 . 0 3 0 9 6}$ & $\mathbf{0 . 0 5 5 6 3}$ & $\mathbf{0 . 0 3 4 8 1}$ \\
KinectFusion & 22.37200 & 0.24311 & 2.99067 & 3.85941 & 0.11136 \\
Go-ICP & nan & 0.825212 & 0.01832 & 0.358507 & $\mathbf{0 . 0 0 8 8 3}$ & 0.09836 \\
\hline
\end{tabular}

The boldface entries are for emphasis for better result in comparison between the new method with other methods 
Table 3 Results of Chess, Fire, Heads, and Office datasets

\begin{tabular}{|c|c|c|c|c|c|}
\hline Scene name & Algorithm & Min & Max & Mean & St. dev. \\
\hline Chess & ISADE & 0.10047 & 0.11187 & 0.10230 & 0.002821482 \\
\hline KinectFusion & $\mathrm{DE}$ & 0.17453 & 3.92808 & 0.29860 & 0.112087291 \\
\hline \multirow[t]{3}{*}{ ref: 22.372} & $\mathrm{GA}$ & 1.44923 & 1.80180 & 2.53723 & 0.691936150 \\
\hline & SA & 1.11736 & 2.55157 & 1.65871 & 0.400817542 \\
\hline & PSO & 1.19899 & 2.58186 & 1.72316 & 0.459892382 \\
\hline Fire & ISADE & 0.03169 & 0.03196 & 0.03179 & $8.70855 E-005$ \\
\hline KinectFusion & $\mathrm{DE}$ & 0.03873 & 0.26059 & 0.10263 & 0.066038287 \\
\hline \multirow[t]{3}{*}{ ref: 0.243112} & GA & 0.22177 & 3.93133 & 1.58268 & 0.913837133 \\
\hline & SA & 0.15060 & 0.88670 & 0.45855 & 0.249700426 \\
\hline & PSO & 0.11158 & 0.63419 & 0.34592 & 0.151824890 \\
\hline Heads & ISADE & 0.00994 & 0.01016 & 0.01000 & $7.01799 E-005$ \\
\hline KinectFusion & $\mathrm{DE}$ & 0.01276 & 0.06570 & 0.02205 & 0.012768061 \\
\hline \multirow[t]{3}{*}{ ref: 2.99067} & GA & 0.47056 & 1.70316 & 0.97758 & 0.358190303 \\
\hline & SA & 0.30740 & 1.01428 & 0.65404 & 0.264058658 \\
\hline & PSO & 0.20801 & 1.88772 & 0.54401 & 0.463097716 \\
\hline Office & ISADE & 0.03084 & 0.03115 & 0.03096 & $8.39925 E-005$ \\
\hline KinectFusion & $\mathrm{DE}$ & 0.03195 & 0.06436 & 0.04373 & 0.009462166 \\
\hline \multirow[t]{3}{*}{ ref: 3.85941} & GA & 0.24518 & 4.05346 & 1.88819 & 0.928751342 \\
\hline & SA & 0.10385 & 2.67972 & 0.84426 & 0.720046753 \\
\hline & PSO & 0.07169 & 2.08078 & 0.58507 & 0.686244921 \\
\hline
\end{tabular}

The boldface entries are for emphasis for better result in comparison between the new method with other methods

$\mathrm{DE}$, where the horizontal axis represents the iteration, and the vertical axis represents the error. In comparison with ISADE, DE required significant larger iteration number to achieve convergence. With ISADE, from 70 iterations, all the results show a flat trend and no new optimal solutions with a significant difference are found. This iteration number for $\mathrm{DE}$ is 120.

These results show that, if we reduce the maximum number of iterations to 70 , the results remain the same.

Table 4 Results of Pumpkin, RedKitchen and Stairs datasets

\begin{tabular}{|c|c|c|c|c|c|}
\hline Scene name & Algorithm & Min & Max & Mean & St. dev. \\
\hline Pumpkin & ISADE & 0.05541 & 0.05603 & 0.05563 & 0.000175987 \\
\hline KinectFusion & $\mathrm{DE}$ & 0.06555 & 0.16927 & 0.11105 & 0.111050113 \\
\hline \multirow[t]{3}{*}{ ref: 0.111361} & GA & 0.45803 & 3.15529 & 1.42922 & 0.775060060 \\
\hline & SA & 0.07468 & 0.90335 & 0.49504 & 0.248322702 \\
\hline & PSO & 0.11181 & 1.43345 & 0.36443 & 0.334116975 \\
\hline RedKitchen & ISADE & 0.03423 & 0.03759 & 0.03481 & 0.000915588 \\
\hline KinectFusion & DE & 0.05879 & 0.60304 & 0.17479 & 0.149183155 \\
\hline \multirow[t]{3}{*}{ ref: 0.0983645} & SA & 0.52141 & 5.48133 & 2.07233 & 1.339500137 \\
\hline & GA & 0.12508 & 1.58015 & 0.62601 & 0.441544434 \\
\hline & PSO & 0.05515 & 2.48188 & 0.54354 & 0.671268667 \\
\hline Stairs & ISADE & 0.00875 & 0.00898 & 0.00883 & 0.000079463 \\
\hline KinectFusion & $\mathrm{DE}$ & 0.00975 & 0.04665 & 0.01767 & 0.009514675 \\
\hline \multirow[t]{3}{*}{ ref: 0.0156084} & SA & 0.21207 & 2.24988 & 1.19252 & 0.627554990 \\
\hline & GA & 0.01405 & 1.08881 & 0.29528 & 0.304574563 \\
\hline & PSO & 0.04632 & 0.96723 & 0.25021 & 0.239971819 \\
\hline
\end{tabular}

The boldface entries are for emphasis for better result in comparison between the new method with other methods 

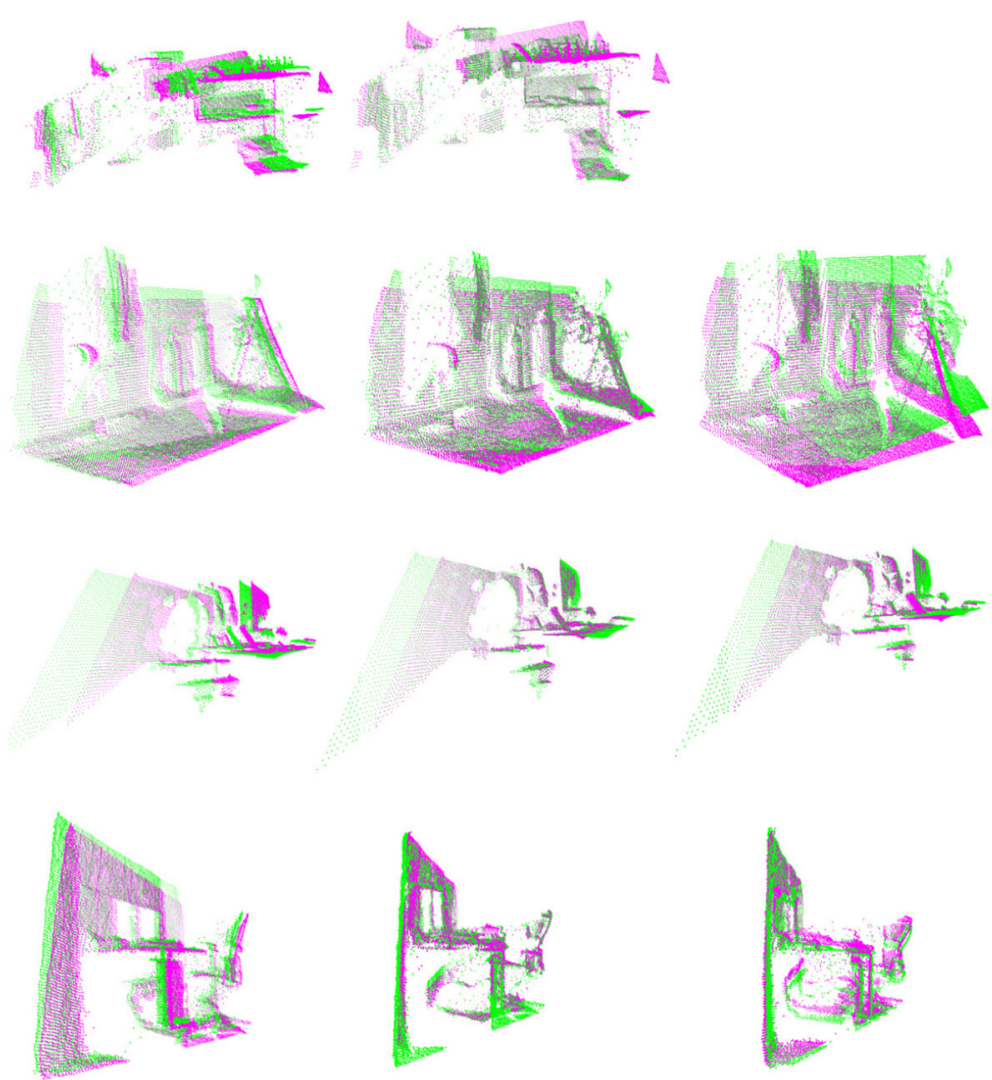

Fig. 9 First four scenes (Chess, Fire, Heads, Office) registration output example. KinectFusion results are in the left hand side, the new algorithm's results are in the center, and Go-ICP algorithm's results are on the right hand side
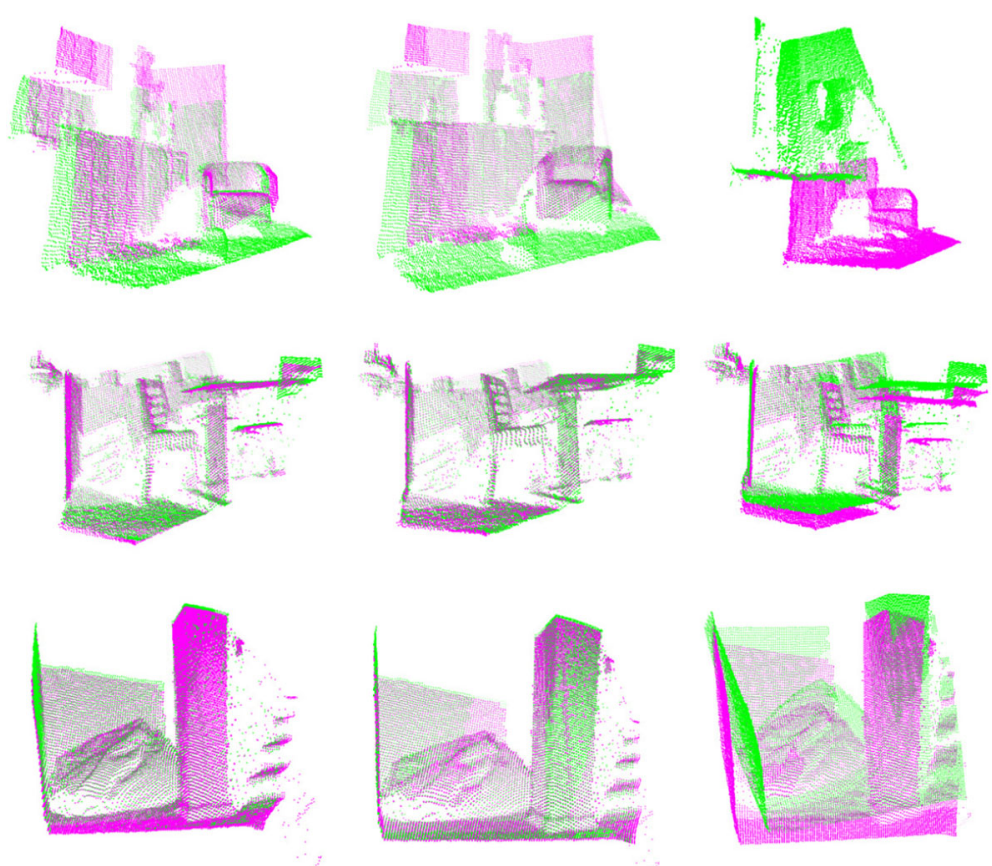

Fig. 10 Last three scenes (Pumpkin, RedKitchen, Stairs) registration output example. KinectFusion results are in the left hand side, the new algorithm's results are in the center, and Go-ICP algorithm's results are on the right hand side 

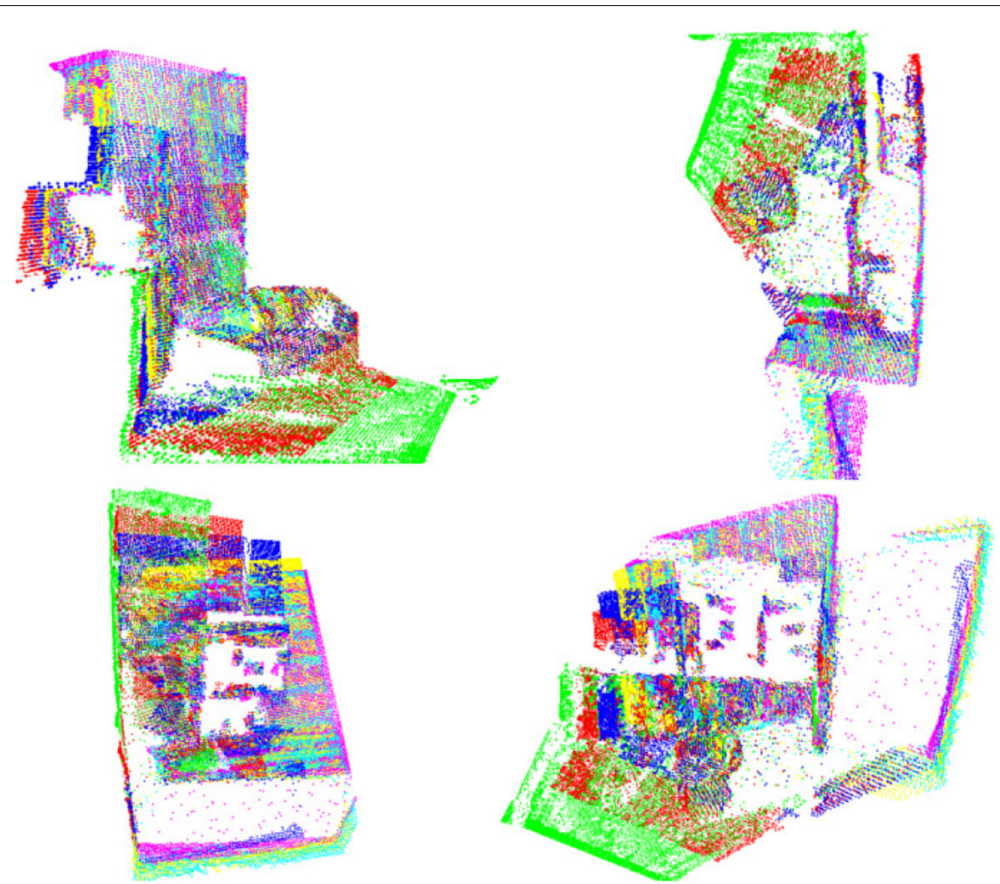

Fig. 11 Office scene reconstructed results from different view angles

Clearly, the smaller the iteration number, the shorter is the runtime.

\subsection{Results from registering in different movement patterns and frame distances}

Figure 13 shows the values of rotation angles $(\alpha, \beta, \gamma)$ in radian and translation distances $(x, y, z)$ in meter of $3 \mathrm{D}$ camera movement. Those values were obtained by using new algorithm to register range images from frame 001 to 060 respectively into the frame 000 of seq- 01 in different datasets. The process stops if the movement values get over searching boundaries. From all datasets, we choose three typical movement of Chess, Fire, and Heads datasets for rotating, sliding, and forwarding with rotating movements respectively.

The results with no sudden value changing between two consecutive frames verify the feasibility of applying the new algorithm in registering range images of different movement patterns and frame distances.

\subsection{Runtime}

For the data of $128 \times 96$ resolution, average runtime for the proposed method are shown in Table 5. In the results, the average runtime for registration is around $0.6 \mathrm{~s}$ for 150 iterations of all scenes. Since the distance between two frames is 20 , the registering equivalence rate is 33 frames

Table 5 Average running time (in second) on different scenes of new methods and Go-ICP

\begin{tabular}{|c|c|c|c|c|}
\hline & $\begin{array}{l}\text { New methods } \\
100 \text { generations }\end{array}$ & $\begin{array}{l}\text { New method } \\
150 \text { generations }\end{array}$ & $\begin{array}{l}\text { Go-ICP } \\
\text { distTransSize = } 50\end{array}$ & $\begin{array}{l}\text { Go-ICP } \\
\text { distTransSize }=100\end{array}$ \\
\hline Chess & 0.388414 & 0.516832 & inf & inf \\
\hline Fire & 0.385928 & 0.625765 & 14.2786 & inf \\
\hline Heads & 0.335828 & 0.562451 & 0.102944 & 0.104659 \\
\hline Office & 0.378768 & 0.560734 & 0.030326 & 34.411 \\
\hline Pumpkin & 0.410615 & 0.621756 & 104.468 & inf \\
\hline RedKitchen & 0.415258 & 0.588466 & 30.3815 & inf \\
\hline Stairs & 0.409834 & 0.597050 & 188.205 & inf \\
\hline
\end{tabular}




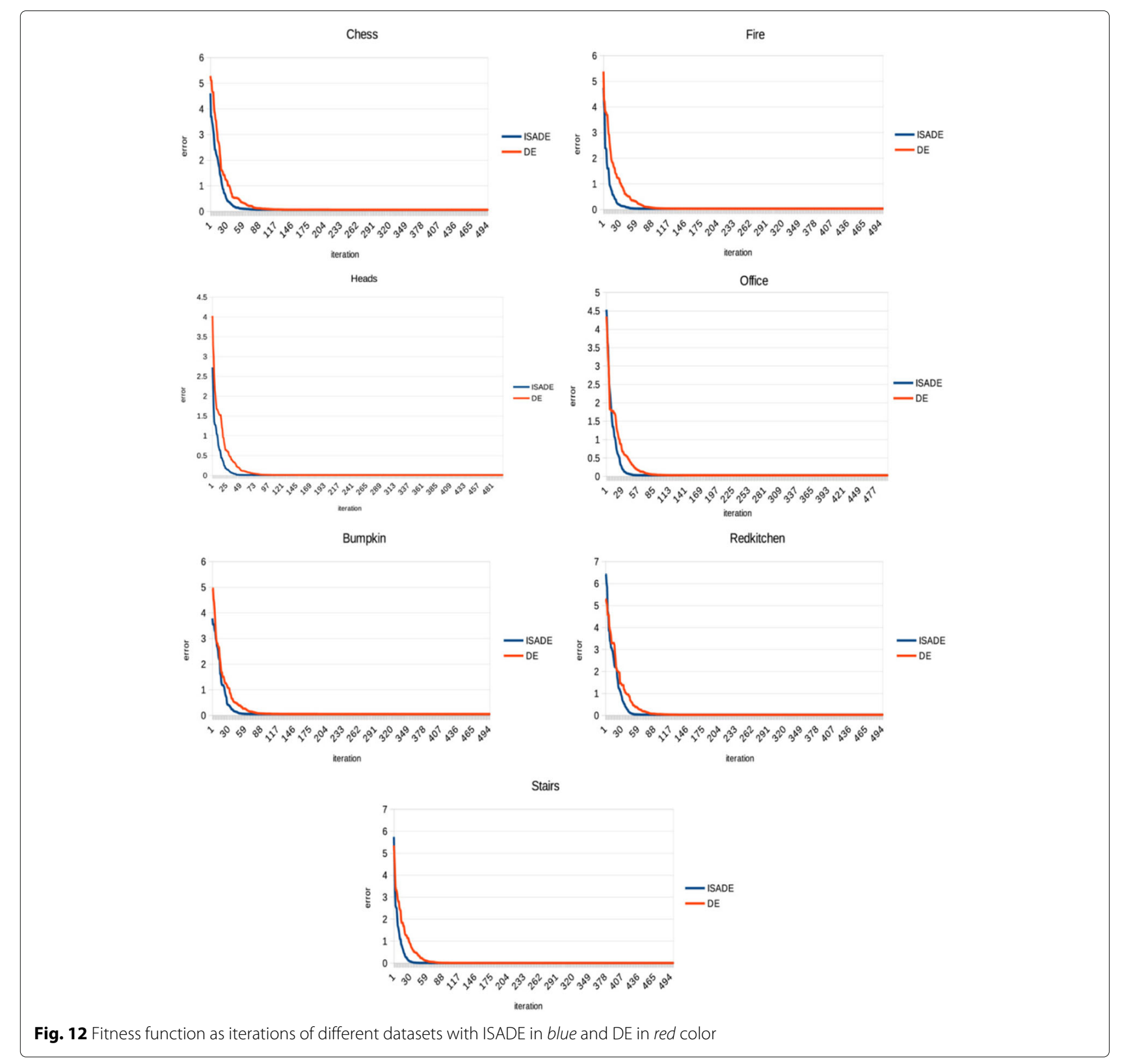

per second (fps). At this rate, when we move the camera, the algorithm are able to update the scenarios.

By subsampling the data range image and remaining the model range image, the new algorithm gain smaller runtime while error level stays unchanged. Figure 14 shows the runtime at different level of subsample on the right hand and the errors in the left hand for the RedKitchen scenario.

\section{Discussion}

Image registration has become a very active research area. Recently, the approach of using EAs, in particular in new methods, proved their potential for tack- ling the image registration problem based on their robustness and accuracy for searching for global optimal solutions. When EAs are used as search tools, good initial conditions are not necessary for avoiding local minima while converging to near-global minima solutions.

We proposed a novel registration method in which a fast ray-casting-based error calculation is integrated with a powerful self-adaptive optimization algorithm. The experimental results showed that ISADE is able to find a robust and accurate transformation matrix, while the ray-casting method is fast and efficient in calculating error for global registration problems. 


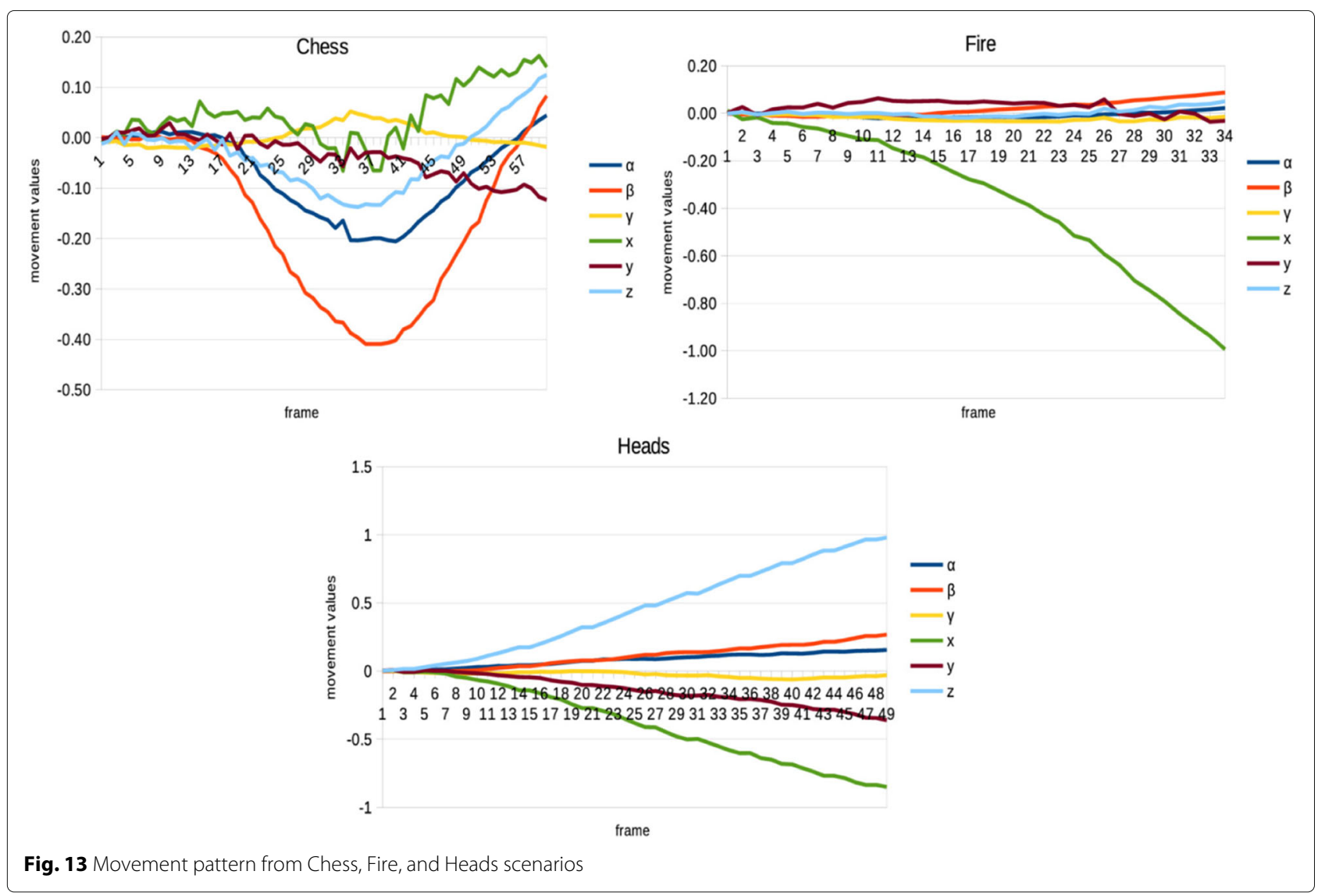

A more important point is that, by eliminating inner ICP loops in hybrid integrations and fine-tuning procedures applied in previously proposed methods, the newly proposed method becomes the first direct, as well as the first online potential, global registration algorithm. Its robustness and accuracy were tested and verified in real 3D scenes captured by a Microsoft Kinect camera.
Currently, the algorithm is implemented using a CPU parallel procedure. In future work, the new algorithm can be implemented on a GPU to reduce its runtime and error while retaining its accuracy and robustness. Furthermore, the method can be extended for general point clouds from different sources by using a virtual camera surface and presenting it as a constructed surface. The proposed
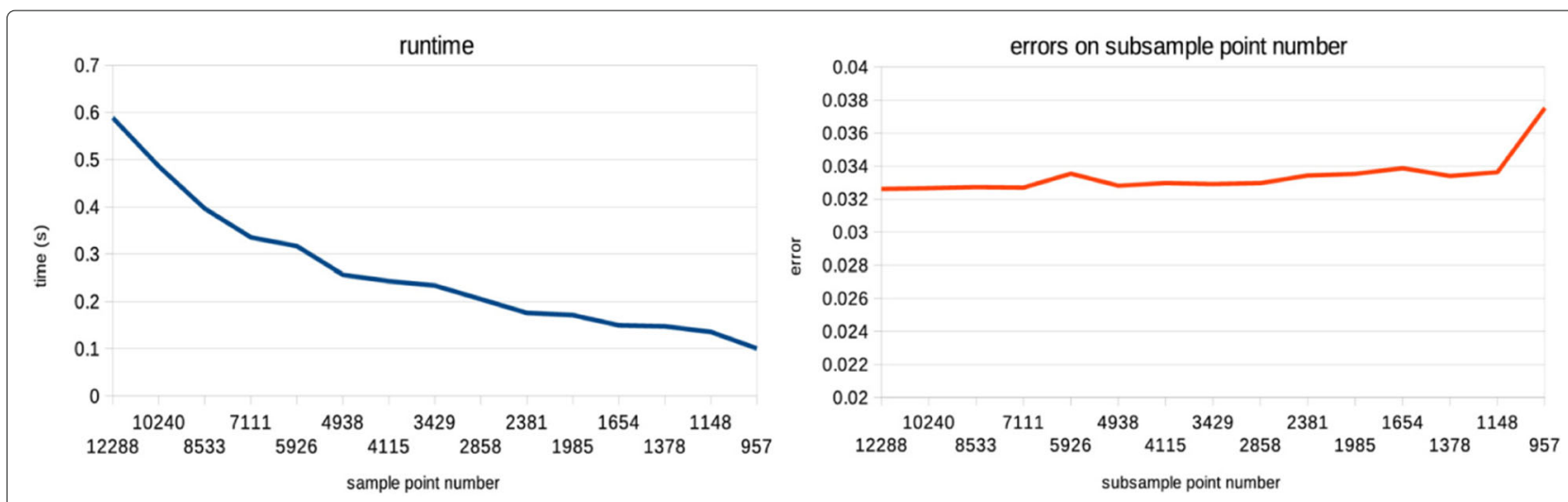

Fig. 14 Runtime and error on subsample point numbers 
method is also potentially suitable for super resolution range images.

\section{Authors' contributions}

LT took charge of the system coding, doing experiments, data analysis and writing the whole paper excluding ISADE algorithm part at Subsection 3.3. TB took charge of coding and writing for ISADE algorithm part at Subsection 3.3. $\mathrm{HH}$ took charge of advisor position for paper presentation, experiment design, data analysis presentation as well English revising. All authors read and approved the final manuscript.

\section{Competing interests}

The authors declare that they have no competing interests.

\section{Publisher's Note}

Springer Nature remains neutral with regard to jurisdictional claims in published maps and institutional affiliations.

\section{Author details}

${ }^{1}$ Department of Functional Control System, Shibaura Institute of Technology, 307 Fukasaku, Minuma-ku, Saitama City, Saitama, 337-8570, Japan. ${ }^{2}$ School of Mechanical Engineering, Hanoi University of Science and Technology, 1 Dai Co Viet Road, Ha Noi, Viet Nam. ${ }^{3}$ Department of Machinery and Control System, Shibaura Institute of Technology, 307 Fukasaku, Minuma-ku, Saitama City, Saitama, 337-8570, Japan.

\section{Received: 29 March 2016 Accepted: 12 April 2017}

Published online: 08 May 2017

\section{References}

1. Sharp GC, Lee SW, Wehe DK (2004) Multiview registration of 3D scenes by minimizing error between coordinate frames. IEEE Trans Pattern Anal Mach Intell 26(8):1037-1050. doi:10.1109/TPAMI.2004.49

2. Holz D, Ichim AE, Tombari F, Rusu RB, Behnke S (2015) Registration with the Point Cloud Library: a modular framework for aligning in 3-D. IEEE Robot Autom Mag 22(4):110-124. doi:10.1109/MRA.2015.243233

3. Besl P, McKay ND (1992) A method for registration of 3-d shapes. IEEE Trans Pattern Anal Mach Intell 14(2):239-256. doi:10.1109/34.121791

4. Granger S, Pennec X (2002) Multi-scale EM-ICP: a fast and robust approach for surface registration. In: European Conference on Computer Vision, vol. 2353. pp 418-432

5. Segal A, Haehnel D, Thrun S (2009) Generalized-ICP. In: Proceedings of Robotics: Science and Systems, Seattle, USA. doi:10.15607/RSS.2009.V.021

6. Meshlab. http://meshlab.sourceforge.net/. Accessed 15 Jan 2017

7. Rusu RB, Marton ZC, Blodow N, Beetz M (2008) Persistent point feature histograms for 3D point clouds. In: Proceedings of the 10th International Conference on Intelligent Autonomous Systems (IAS-10), Baden-Baden. pp 119-128

8. Sehgal A, Cernea D, Makaveeva M (2010) Real-time scale invariant 3D range point cloud registration. In: Campilho A, Kamel M (eds). Image Analysis and Recognition. ICIAR 2010. Lecture Notes in Computer Science. Springer, Berlin Heidelberg Vol. 6111. doi:10.1007/978-3-642-13772-3_23

9. Wu F, Fang X (2007) An improved RANSAC homography algorithm for feature based image mosaic. In: Proceedings of the 7th WSEAS International Conference on Signal Processing, Computational Geometry \& Artificial Vision, ISCGAV'07, World Scientific and Engineering Academy and Society (WSEAS), Stevens Point, Wisconsin. pp 202-207. http://dl. acm.org/citation. cfm?id=1364592.1364627

10. Yang J, Li H, Jia Y (2013) Go-icp: Solving 3D registration efficiently and globally optimally. In: 2013 IEEE International Conference on Computer Vision, Sydney. pp 1457-1464. doi:10.1109/ICCV.2013.184

11. Luck J, Little C, Hoff W (2000) Registration of range data using a hybrid simulated annealing and iterative closest point algorithm. In: Proceedings 2000 ICRA. Millennium Conference. IEEE International Conference on Robotics and Automation. Symposia Proceedings (Cat. No.00CH37065), San Francisco Vol. 4. pp 3739-3744. doi:10.1109/ROBOT.2000.845314

12. Neumann D, Lugauer F, Bauer S, Wasza J, Hornegger J (2011) Real-time RGB-d mapping and 3-D modeling on the GPU using the random ball cover data structure. In: 2011 IEEE International Conference on Computer Vision Workshops (ICCV Workshops), Barcelona. pp 1161-1167. doi:10.1109/ICCW.2011.6130381
13. Ingber $L$ (1993) Simulated annealing: practice versus theory. Math Comput Model 18(11):29-57. http://dx.doi.org/10.1016/0895-7177(93)90204-C

14. Bui T, Pham H, Hasegawa H (2013) Improve self-adaptive control parameters in differential evolution for solving constrained engineering optimization problems. J Comput Sci Technol 7(1):59-74. doi:10.1299/jcst.7.59

15. Marden S, Guivant J (2012) Improving the performance of ICP for real-time applications using an approximate nearest neighbour search. In: Proceedings of Australasian Conference on Robotics and Automation, New Zealand. pp 3-5

16. Lim Low K (2004) Linear least-squares optimization for point-toplane ICP surface registration. Tech Rep TR04-004, Department of Computer Science, University of North Carolina at Chapel Hill

17. Bouaziz S, Tagliasacchi A, Pauly M (2013) Sparse iterative closest point. In: Proceedings of the Eleventh Eurographics/ACMSIGGRAPH Symposium on Geometry Processing, SGP '13, Eurographics Association. Aire-la-Ville, Switzerland. pp 113-123. doi:10.1111/cgf.12178

18. Izadi S, Kim D, Hilliges O, Molyneaux D, Newcombe R, Kohli P, Shotton J, Hodges S, Freeman D, Davison A, Fitzgibbon A (2011) Kinect-Fusion: real-time $3 \mathrm{~d}$ reconstruction and interaction using a moving depth camera. In: Proceedings of the 24th Annual ACM Symposium on User Interface Software and Technology, UIST '11. ACM, New York. pp 559-568. doi:10.1145/2047196.2047270

19. Chandran S (2012) Introduction to kd-trees. University of Maryland Department of Computer Science. https://www.cs.umd.edu/class/ spring2002/cmsc420-0401/pbasic.pdf

20. Roth SD (1982) Ray casting for modeling solids. Comput Graph Image Process 18(2):109-144. doi:http://dx.doi.org/10.1016/0146-664X(82)90169-1

21. Point cloud library. http://pointclouds.org/. Accessed 15 Jan 2017

22. Openmp. http://openmp.org/wp/. Accessed: 15 Jan 2017

23. Storn R, Price K (1997) Differential evolution a simple and efficient heuristic for global optimization over continuous spaces. J Glob Optim 11(4):341-359. doi:10.1023/A:1008202821328

24. Tooyama S, Hasegawa H (2009) Adaptive plan system with genetic algorithm using the variable neighborhood range control. In: Evolutionary Computation, 2009. CEC '09. IEEE Congress on Evolutionary Computation, CEC '09. pp 846-853. doi:10.1109/CEC.2009.4983033

25. Chen YW, Mimori A, Lin C-L (2009) Hybrid particle swarm optimization for 3-d image registration. In: 16th IEEE International Conference on Image Processing (ICIP), Cairo. pp 1753-1756. doi:10.1109/ICIP.2009.5414613

26. Seixas FL, Ochi LS, Conci A, Saade DM (2008) Image registration using genetic algorithms. In: Proceedings of the 10th Annual Conference on Genetic and Evolutionary Computation, GECCO '08. ACM, New York. pp 1145-1146. doi:10.1145/1389095.1389320

27. Falco ID, Cioppa AD, Maisto D, Tarantino E (2008) Differential evolution as a viable tool for satellite image registration. Appl Soft Comput 8(4):1453-1462. Soft Computing for Dynamic Data Mining. doi:http://dx. doi.org/10.1016/j.asoc.2007.10.013

28. Iterative closest point implementation C++ code. http://www.cvlibs.net/ software/libicp/. Accessed 15 Jan 2017

29. Go-ICP implementation C++ code. http://iitlab.bit.edu.cn/mcislab/ yangjiaolong/go-icp/. Accessed 15 Jan 2017 